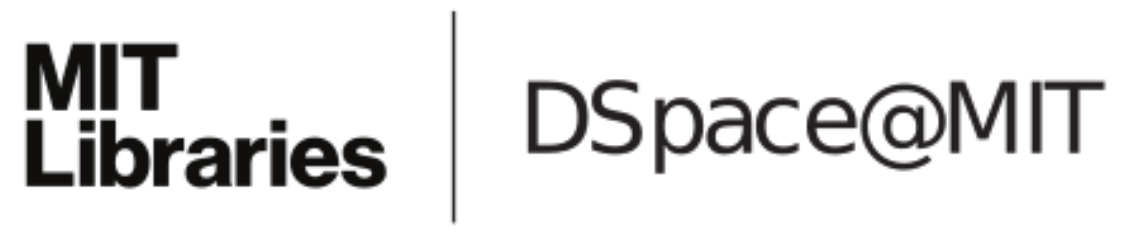

\author{
MIT Open Access Articles
}

Existence of minimal surfaces of arbitrarily large Morse index

The MIT Faculty has made this article openly available. Please share how this access benefits you. Your story matters.

Citation: Li, Haozhao, and Xin Zhou. “Existence of Minimal Surfaces of Arbitrarily Large Morse Index." Calculus of Variations and Partial Differential Equations 55.3 (2016): n. pag.

As Published: http://dx.doi.org/10.1007/s00526-016-1007-6

Publisher: Springer Berlin Heidelberg

Persistent URL: http://hdl.handle.net/1721.1/107182

Version: Author's final manuscript: final author's manuscript post peer review, without publisher's formatting or copy editing

Terms of Use: Article is made available in accordance with the publisher's policy and may be subject to US copyright law. Please refer to the publisher's site for terms of use. 


\title{
Existence of minimal surfaces of arbitrarily large Morse index
}

\author{
Haozhao Li ${ }^{1} \cdot$ Xin Zhou ${ }^{2}$
}

Received: 7 April 2015 / Accepted: 11 April 2016 / Published online: 21 May 2016

(C) Springer-Verlag Berlin Heidelberg 2016

\begin{abstract}
We show that in a closed 3-manifold with a generic metric of positive Ricci curvature, there are minimal surfaces of arbitrary large Morse index, which partially confirms a conjecture by Marques and Neves. We prove this by analyzing the lamination structure of the limit of minimal surfaces with bounded Morse index.
\end{abstract}

Mathematics Subject Classification 53A10 - 49Q05 - 58E12 - 53C42

\section{Introduction}

In 1982, Yau conjectured that every closed 3-manifold admits infinitely many smooth, closed, immersed minimal surfaces [36, Problem 88]. This was recently confirmed by Marques and Neves when the ambient manifold has positive Ricci curvature [22]; they proved the existence of infinitely many smooth, closed, embedded minimal hypersurfaces in manifold $M^{n+1}$ of positive Ricci curvature when $2 \leq n \leq 6$. They [21,22,25] also conjectured that generically the Morse indices of these minimal hypersurfaces should grow linearly to infinity. However, it is even unknown that whether the Morse indices of these minimal surfaces are bounded.

Communicated by A. Neves.

The author is partially supported by NSFC Grant No. 11131007. The author is partially supported by NSF Grant DMS-1406337.

$凶 \quad$ Xin Zhou

xinzhou@math.mit.edu

Haozhao Li

hzli@ustc.edu.cn

1 Key Laboratory of Wu Wen-Tsun Mathematics, Chinese Academy of Sciences, School of Mathematical Sciences, University of Science and Technology of China, No. 96 Jinzhai Road, Hefei, Anhui Province 230026, China

2 Department of Mathematics, Massachusetts Institute of Technology, 77 Massachusetts Avenue, Cambridge, MA 02139, USA 
In this paper, we address this question in dimension 3 by showing that generically the Morse indices of these minimal surfaces must grow up to infinity.

Denote $M^{3}$ by a 3-manifold. A metric $g$ on $M$ is called bumpy, if any closed embedded minimal surface has no nontrivial Jacobi fields. In [34], White showed that bumpy metrics are generic. Our main result is the following:

Theorem 1.1 Let $M^{3}$ be a closed 3-manifold with a bumpy metric $g$ of positive Ricci curvature. Then there exist embedded minimal surfaces of arbitrarily large Morse index in $\left(M^{3}, g\right)$.

The problem of finding minimal surfaces with fixed topology and arbitrarily large Morse index in 3-manifolds has attracted a lot of interests. This problem originated from a well-known question of Pitts and Rubinstein (c.f. [8,27]). Motivated by this question, Hass-Norbury-Rubinstein [18] constructed a smooth metric on any 3-manifold which admits embedded genus zero minimal surfaces of arbitrarily high Morse index. Colding-Hingston [6] and Colding-De Lellis [5] extended the construction (by different methods), respectively, to find metrics on any 3-manifold which admits minimal surfaces with no Morse index bound of genus 1 and genus $\geq 2$. Compared to these results, our result gives a large family of metrics on 3-manifold which admit minimal surfaces of arbitrarily large Morse index, while assuming no genus bound.

In fact, Theorem 1.1 is a direct corollary of the following finiteness theorem for minimal surfaces of bounded Morse index in a bumpy metric and Marques-Neves's result in [22]. Let $M^{3}$ be a closed 3-manifold with a bumpy metric $g$ of positive Ricci curvature, and $\mathcal{M}$ the space of embedded, closed, minimal surfaces in $(M, g)$. By [22], $\mathcal{M}$ contains infinitely many elements. Given $N \gg 1$, we denote $\mathcal{M}_{N}$ by

$$
\mathcal{M}_{N}=\{\Sigma \in \mathcal{M}: \quad \operatorname{Ind}(\Sigma) \leq N\} .
$$

Then we have

Theorem 1.2 Let $M^{3}$ be a closed 3-manifold with a bumpy metric $g$ of positive Ricci curvature. Then there are only finitely many elements in $\mathcal{M}_{N}$.

Remark 1.3 The result is not true in general if we remove the assumption of positive Ricci curvature, as Colding and Minicozzi [7] constructed an open set of metrics on any compact 3 -manifolds which admit stable embedded minimal tori of arbitrarily large area ${ }^{1}$.

Theorem 1.2 is proved by studying the limit of a sequence of minimal surfaces of bounded Morse index. Convergence theorems for minimal surfaces have been studied extensively in the past, especially in 3-dimension. Choi-Schoen [3] proved the compactness of embedded minimal surfaces of fixed topological type in a closed 3-manifold with positive Ricci curvature. Anderson [1] and White [33] respectively proved similar convergence and compactness results for minimal surfaces and surfaces that are stationary for parametric elliptic functionals in 3-manifold with bounded genus and bounded area. For higher dimensional case, in a recent work [29], Sharp showed a convergence result for minimal hypersurfaces with bounded Morse index and bounded volume. A key technical condition of these results is the area bound. Without this assumption, Colding and Minicozzi developed a whole theory [9-13] to study the convergence of minimal surfaces with fixed genus but no area bound. The key point in Colding-Minicozzi's theory is to study the lamination convergence of minimal surfaces. Our proof of Theorem 1.2 utilizes this idea, and the convergence result in the proof

$\overline{1 \text { We would like to thank the referee for pointing out this example to us. }}$ 
can be thought as an analogy of Colding-Minicozzi's theory for minimal surfaces with fixed Morse index but no area bound.

At the end, we want to mention Colding-Minicozzi's finiteness theorem [8] and a corollary.

Theorem 1.4 (Colding-Minicozzi [8, Theorem1.1]) Let $M^{3}$ be a closed orientable 3manifold with a bumpy metric $g$, and $V$, e fixed. There exist at most finitely many closed embedded minimal surfaces in $M$ with genus $e$ and area at most $V$.

By Yang-Yau [35] and Choi-Wang [4], in a Riemmannian 3-manifold $M$ with Ric $c_{g} \geq \Lambda>0$, any closed embedded minimal surface with fixed genus $e$ has area bounded from above by $\frac{16 \pi(1+e)}{\Lambda}$ (see also [14, §7.5]). Combining with Marques-Neves’s result [22], we have:

Corollary 1.5 Let $M^{3}$ be a closed 3-manifold with a bumpy metric $g$ of positive Ricci curvature. Then there exist embedded minimal surfaces of arbitrarily large genus in $\left(M^{3}, g\right)$.

We sketch the proof of Theorem 1.2 as follows. If Theorem 1.2 were not true, let $\left\{\Sigma_{i}\right\}$ be a sequence in $\mathcal{M}_{N}$ (1.1). The Morse index bound implies that $\Sigma_{i}$ are locally stable away from finitely many points, and hence converge locally smoothly to a minimal lamination $\mathcal{L}$ outside these points by Schoen's curvature estimates [28]. Assume that $\mathcal{L}$ is orientable and consider the leaves of $\mathcal{L}$. If there is no isolated leaf, then we can construct a positive Jacobi field along an accumulating leaf $\Lambda$, and hence show that $\Lambda$ has only removable singularities; so its closure $\bar{\Lambda}$ is a smooth, embedded, stable minimal surface, which contradicts the positive Ricci curvature condition.

If $\mathcal{L}$ has an isolated leaf $\Lambda$ for which the multiplicity of the convergence of $\Sigma_{i}$ to $\Lambda$ is infinite or greater than one, then we can also construct a positive Jacobi field, and hence get a contradiction by using the same argument as above. In the case of multiplicity one convergence, we show that the leaf must be proper, then we construct a nontrivial Jacobi vector field which extends across the singularities, hence contradicting the bumpy condition. One main technical difficulty here is the fact that $\Sigma_{i}$ might not a priori converge in the sense of varifold. For the case that $\mathcal{L}$ is non-orientable, we can get a contradiction by combining the above arguments with the structure of non-orientable hypersurfaces (c.f. [37, §3]).

\section{Preliminaries}

In this section, we recall some basic facts on minimal surfaces in 3-manifolds. Let $M^{3}$ be a closed 3-manifold with a Riemannian metric $g$. A minimal surface $\Sigma^{2} \subset M^{3}$ is a critical point for the area functional, or equivalently a smooth surface with mean curvature equal to zero. $\Sigma$ is called two-sided, if $\Sigma$ has trivial normal bundle, i.e. there exists a unit normal vector field $\nu$. The Jacobi operator for the second variation of the area functional is given by (c.f. $[14, \S 1.8])$

$$
L=\Delta_{\Sigma}+|A|^{2}+R i c_{g}(v, v),
$$

where $\Delta_{\Sigma}$ is the Laplacian operator on $\Sigma$ with the induced metric, and $A$ is the second fundamental form of $\Sigma$, and Ric is the Ricci curvature of $(M, g) . \lambda \in \mathbb{R}$ is an eigenvalue of $L$ if there exists a smooth function $\phi \in C^{\infty}(\Sigma)$ such that $L \phi=-\lambda \phi$.

The Morse index of $\Sigma$, denoted by $\operatorname{Ind}(\Sigma)$, is the number of negative eigenvalues of $L$ counted with multiplicity. $\Sigma$ is called stable if the Morse index is zero. When $R i c_{g}>0$, there is no two-sided, stable, closed or complete, minimal surface. The case for closed minimal surface is trivial by plugging in constant test function in the second variation of area; while 
the case for complete minimal surface is given in the Appendix, c.f. Theorem 4.2. A Jacobi field $\phi v$ on $\Sigma$ is a solution of $L \phi=0$.

For stable minimal surfaces, we recall the curvature estimates due to Schoen [28] (see also [14, Corollary 2.11]).

Theorem 2.1 Given $k>0$, if $\Sigma^{2} \subset B_{r_{0}}(x) \subset M^{3}$ is an immersed stable minimal surface with trivial normal bundle, where the sectional curvature of $K_{M}$ of $M$ satisfies $\left|K_{M}\right| \leq k^{2}$, the radius $r_{0}<\rho_{1}(\pi / k, k)$ and $\partial \Sigma \subset \partial B_{r_{0}}(x)$, then for some $C=C(k)>0$ and all $0<\sigma \leq r_{0}$,

$$
\sup _{B_{r_{0}-\sigma}(x) \cap \Sigma}|A|^{2} \leq \frac{C}{\sigma^{2}} .
$$

Now we introduce the definition of lamination.

Definition 2.2 (cf. Thurston [32], Colding-Minicozzi [8]) A codimension one lamination of $M^{3}$ is a collection $\mathcal{L}$ of smooth, disjoint, connected surfaces, denoted by $\Lambda$ (called leaves) such that $\cup_{\Lambda \in \mathcal{L}} \Lambda$ is closed. Moreover for each $x \in M$ there exist an open neighborhood $U$ of $x$ and a local coordinate chart, $(U, \Phi)$, with $\Phi(U) \subset \mathbb{R}^{3}$ such that in these coordinates the leaves in $\mathcal{L}$ pass through the chart in slices of the form $\left(\mathbb{R}^{2} \times\{t\}\right) \cap \Phi(U)$.

In [12], Colding-Minicozzi discussed the compactness of laminations. Recall that a minimal lamination is a lamination whose leaves are minimal. A sequence of laminations is said to converge if the corresponding coordinate maps converge.

Theorem 2.3 ([12, Proposition B.1]) Let $M^{3}$ be a fixed 3-manifold. If $\mathcal{L}_{i} \subset B_{2 R}(x) \subset M$ is a sequence of minimal laminations with uniformly bounded curvatures (where each leaf has boundary contained in $\partial B_{2 R}(x)$ ), then a subsequence of $\mathcal{L}_{i}$ converges in the $C^{\alpha}$ topology for any $\alpha<1$ to a Lipschitz, lamination $\mathcal{L}$ in $B_{R}(x)$ with minimal leaves.

Remark 2.4 Here the "Lipschitz" regularity means that the coordinate charts of $\mathcal{L}$ are Lipschitz, while each leave is still smooth.

We need the removable singularity theorem for stable minimal surfaces.

Lemma 2.5 Given a 3-manifold $M$ and a point $p \in M$, let $\Sigma \subset M \backslash\{p\}$ be a connected, properly embedded, minimal surface with trivial normal bundle. If $\Sigma$ is stable in a local geodesic ball $B_{r_{0}}(p)$ and $B_{r_{0}}(p) \cap(\bar{\Sigma} \backslash \Sigma)=\{p\}$, then $\Sigma$ has a removable singularity at $p$.

Remark 2.6 Lemma 2.5 is proved for $M=\mathbb{R}^{3}$ by Gulliver-Lawson [20, Theorem 3.3] and Colding-Minicozzi [13, Lemma A.35]. We present a proof based on ideas by ColdingMinicozzi [13, Lemma A.35] and White [33] for completeness. Note that this result also follows by combining [19, Lemma 1] and [29, Remark A.2].

Proof Consider the blow up $\Sigma_{i}=\lambda_{i}^{-1} \Sigma$ (by embedding $M$ into some $\mathbb{R}^{N}$ ) of $\Sigma$ at $p$ by an arbitraty sequence of numbers $\lambda_{i}$ decreasing to 0 . By the scaling invariant curvature estimates (2.2), $\Sigma_{i}$ converge locally smoothly to a complete, stable, minimal surface $\Sigma^{\prime}$ in $\mathbb{R}^{3} \backslash\{0\}$. The Bernstein theorem for stable minimal surfaces with point singularities in $\mathbb{R}^{3}$ (c.f. [20, Corollary3.16][13, LemmaA.34]) implies that $\Sigma^{\prime}$ is a union of planes. By an argument of White [33, pp251], this implies that, for small enough $r$, the distance function $x \rightarrow \operatorname{dist}_{M}(x, p)$ of $M$ when restricted on $\Sigma \cap B_{r}(p)$ has no critical points except for strict local minima, and hence (by Morse theory) each connected component of $\Sigma \cap B_{r}$ is 
either a disk with exactly one critical point, or a punctured disk with no critical point. Take a connected component $\tilde{\Sigma}$ of $\Sigma \cap B_{r}$ that is a punctured disk, then the removable singularity theorem for minimal surface with finite Euler characteristic by Gulliver [19] and Choi-Schoen [3, Proposition1] implies that $\tilde{\Sigma}$ has a removable singularity at $p$. So Maximum Principle implies that $\Sigma \cap B_{r}$ has only one component, and $\left(\Sigma \cap B_{r}\right) \cup\{p\}$ is an embedded minimal disk.

We also need the following removable singularity theorem for minimal laminations with a point singularity.

Theorem 2.7 Give a minimal lamination $\mathcal{L}$ of a punctured ball $B_{r}(p) \backslash\{p\}$ in a 3-dimensional Riemannian manifold $\left(M^{3}, g\right)$, if the leaf is either stable when it is orientable, or has a stable orientable double cover, then $\mathcal{L}$ extends to a minimal lamination of $B_{r}(p)$.

Remark 2.8 This theorem is a direct corollary of the combination of Schoen's curvature estimates (c.f. Theorem 2.1) and the Local Removable Singularity Theorem of Meeks-Pérez-Ros [24, Theorem1.1]. In particular, Schoen's curvature estimates applied to each orientable leave $\Lambda$ (or its orientable double cover when $\Lambda$ is nonorientable) implies that $\left|A_{\Lambda}\right|(x) d_{M}(x, p) \leq C$ for all $x \in \Lambda \subset \mathcal{L}$, and this is the only requirement for $\mathcal{L}$ to apply [24, Theorem1.1].

\section{Proof of Theorem 1.2}

In this section, we prove Theorem 1.2. First, we recall an elementary fact (c.f. Fischer-Colbrie [15, Proposition 1] and Sharp [29, Lemma 3.1]):

Lemma 3.1 Suppose $\Sigma^{n} \subset M^{n+1}$ is a closed, smooth, embedded, minimal hypersurface with Morse index $N$. Given any disjoint collection of $N+1$ open sets $\left\{U_{i}\right\}_{i=1}^{N+1}, U_{i} \subset M$, then $\Sigma$ must be stable in $U_{i}$ for some $1 \leq i \leq N+1$.

The next result is an application of Lemma 3.1.

Lemma 3.2 Given a closed, smooth, embedded, minimal surface $\Sigma^{2} \subset M^{3}$ with index at most $N$, then for any $r>0$ small enough, there exist at most $N$ disjoint balls $\left\{B_{r}\left(p_{i}\right)\right\}_{i=1}^{N}$ of $M^{3}$ such that $\Sigma^{2}$ is stable on any ball $B_{r}(x)$ in $M \backslash \cup_{j=1}^{N} B_{r}\left(p_{i}\right)$.

Proof Suppose that the conclusion were not true. Given any disjoint collection of small balls $\left\{B_{r}\left(p_{i}\right)\right\}_{i=1}^{N}$ of $M^{3}$, we can find a ball $B_{r}\left(x_{1}\right) \subset M \backslash \cup_{j=1}^{N} B_{r}\left(p_{i}\right)$ such that $\Sigma$ is unstable in $B_{r}\left(x_{1}\right)$. By Lemma 3.1 there exists a ball, which we assume to be $B_{r}\left(p_{1}\right)$, such that $\Sigma$ is stable in $B_{r}\left(p_{1}\right)$. We now consider the $N$ balls

$$
\mathcal{S}_{1}:=\left\{B_{r}\left(p_{2}\right), \ldots, B_{r}\left(p_{N}\right), B_{r}\left(x_{1}\right)\right\} .
$$

If the set $\mathcal{S}_{1}$ satisfies the property of the lemma, then we stop. Otherwise, there exists a ball $B_{r}\left(x_{2}\right) \subset M \backslash\left(\cup_{j=2}^{N} B_{r}\left(p_{j}\right) \cup B_{r}\left(x_{1}\right)\right)$ such that $\Sigma$ is unstable on $B_{r}\left(x_{2}\right)$. By Lemma 3.1 there exists a ball, which cannot be $B_{r}\left(x_{1}\right)$ and so we assume to be $B_{r}\left(p_{2}\right)$, such that $\Sigma$ is stable in $B_{r}\left(p_{2}\right)$. Then we consider the $N$ balls

$$
\mathcal{S}_{2}:=\left\{B_{r}\left(p_{3}\right), \ldots, B_{r}\left(p_{N}\right), B_{r}\left(x_{1}\right), B_{r}\left(x_{2}\right)\right\} .
$$

If the set $\mathcal{S}_{2}$ satisfies the property of the lemma, then we stop. Otherwise, we repeat the above arguments. We will either stop at $N$ balls satisfying the property of the lemma, or we get the disjoint collection of balls

$$
\mathcal{S}_{N}:=\left\{B_{r}\left(x_{1}\right), \ldots, B_{r}\left(x_{N}\right)\right\}
$$


Note that $\Sigma$ is unstable in any balls $B_{r}\left(x_{i}\right)$. By Lemma 3.1 again, $\Sigma$ will be stable in any ball $B_{r}(p) \subset M \backslash \cup_{j=1}^{N} B_{r}\left(x_{i}\right)$. The lemma is proved.

Now we are ready to prove Theorem 1.2.

Proof of Theorem. 1.2 We divide the proof into the following steps.

Step 1: By contradiction argument, we assume that $\left(M^{3}, g\right)$ is a closed 3-manifold with a bumpy metric $g$, and $\mathcal{M}_{N}$ contains infinitely many elements. Let $\left\{\Sigma_{i}\right\}$ be a sequence in $\mathcal{M}_{N}$. Fix $r>0$. Then for each $\Sigma_{i}$, by Lemma 3.2 there exist at most $N$ balls $\left\{B_{r}\left(p_{i, j}\right)\right\}_{j=1}^{N}$ of $M$, such that $\Sigma_{i}$ is stable in any ball $B_{r}(p) \subset M \backslash \cup_{j=1}^{N} B_{r}\left(p_{i, j}\right)$. Up to a subsequence $\left\{p_{i, j}\right\}$ converge to $\left\{p_{\infty, j}\right\}$ as points, and hence by shrinking $r$, we have that $\Sigma_{i}$ is stable in any ball $B_{r}(p)$ in $M \backslash \cup_{j=1}^{N} B_{2 r}\left(p_{\infty, j}\right)$ for large $i$. Also by basic differential topology, $\Sigma_{i}$ must separate $B_{r}(p)$ when $B_{r}(p)$ is a topological ball, e.g. when $r$ is less than the injective radius of $(M, g)$, and hence $\Sigma_{i} \cap B_{r}(p)$ is orientable and two-sided. By Theorem 2.1, the curvature is uniformly bounded for any $p \in M$ such that $B_{r}(p) \subset M \backslash \cup_{j=1}^{N} B_{2 r}\left(p_{\infty, j}\right)$, i.e.

$$
\sup _{B_{r-\sigma}(p) \cap \Sigma_{i}}\left|A_{\Sigma_{i}}\right|^{2} \leq \frac{C}{\sigma^{2}} .
$$

Therefore, a subsequence of $\left\{\Sigma_{i}\right\}$ (clearly each $\Sigma_{i}$ is a minimal lamination) converges on such a ball $B_{r / 2}(p)$ to a minimal lamination by Theorem 2.3. By passing to further subsequences (still using the same notation), $\left\{\Sigma_{i}\right\}$ converge to a minimal lamination on $M \backslash \cup_{j=1}^{N} B_{2 r}\left(p_{\infty, j}\right)$. Letting $r \rightarrow 0$, then a further subsequence of $\left\{\Sigma_{i}\right\}$ will converge to a minimal lamination of smooth, embedded, disjoint, minimal surfaces $\mathcal{L}=\cup_{\Lambda \in \mathcal{L}} \Lambda$ in $M \backslash\left\{p_{\infty, 1}, \ldots, p_{\infty, N}\right\}$. Therefore, the leaves of $\mathcal{L}$ have at most $N$ singular points. Furthermore, the convergence of $\Sigma_{i}$ to each leaf in $\mathcal{L}$ is locally smooth in $M \backslash\left\{p_{\infty, 1}, \ldots, p_{\infty, N}\right\}$ by the curvature estimates, and hence the leaves of $\mathcal{L}$ satisfy similar local curvature estimates (3.1) as well.

By the Maximum Principle for minimal surfaces [14, Corollary1.28], different sheets can touch at most on the singular sets. We say a leaf $\Lambda$ is isolated if given any compact subset $K \subset \Lambda$, there exists a tubular neighborhood $U$ of $K$ in $M$, such that the intersection of any other leaf with $U$ is empty. Given a leaf $\Lambda \in \mathcal{L}$, then either $\Lambda$ is isolated, or by the curvature estimates (3.1) and the Maximum Principle [14, Corollary1.28], $\Lambda$ must be an accumulating leaf, i.e. there exists a sequence of leaves $\left\{\Lambda_{i}\right\}$ in $\mathcal{L}$, such that $\Lambda_{i}$ converge to $\Lambda$ locally smoothly (with multiplicity one by the definition of lamination).

Step 2: Suppose that there is no isolated leaf. Then pick up an arbitrary accumulating leaf $\Lambda$. Let us first assume $\Lambda$ is orientable. Denote $v$ by the unit normal vector field along $\Lambda$.

We claim that $\Lambda$ is stable. Let $\Lambda_{i}$ be the sequence of leafs in $\mathcal{L}$ which converge to $\Lambda$. The fact that $\Lambda_{i}$ are disjoint from $\Lambda$ implies that possibly up to a subsequence $\Lambda_{i}$ converge to $\Lambda$ locally smoothly from one side. Here convergence from one-side can be explained as follows: given any local ball $B_{r}(p)$ such that the intersection $\Lambda \cap B_{r}(p)$ is diffeomorphic to a disk, and that $\Lambda$ separates $B_{r}(p)$ into two connected components $U_{1}$ and $U_{2}$ with $v$ pointing into $U_{1}$, then for $i$ large all the intersections $\Lambda_{i} \cap B_{r}(p)$ must lie in $U_{1}$. For $i$ large enough we can choose domains $\Omega_{i} \subset \Lambda$ exhausting $\Lambda$ so that each $\Lambda_{i}$ can be written as a normal exponential graph over $\Omega_{i}$ of a function $w_{i}$. Furthermore, we can assume that the graphical functions $w_{i}$ are positive, i.e. $w_{i}>0$, as $\Lambda_{i}$ lies in one-side of $\Lambda$. Denote $u_{i}=w_{i} / w_{i}(p)$ for some fixed $p \in \Lambda$. Then following similar argument as in [8, LemmaA.1], $u_{i}$ converge to a positive solution $u>0$ of the Jacobi equation $L u=0$ on $\Lambda$. By [14, Lemma1.36], the existence of a positive solution $L u=0$ implies that $\Lambda$ is stable. 
If $\Lambda$ is non-orientable, we can consider the orientable double cover $\pi: \tilde{\Lambda} \rightarrow \Lambda$. We claim that $\tilde{\Lambda}$ is a stable immersed minimal surface. In fact, we can consider the pullback of normal bundle $\pi^{*}(v(\Lambda))$, where $v(\Lambda)$ is the normal bundle of $\Lambda$ inside $(M, g)$. Now $\pi^{*}(v(\Lambda))$ forms a double cover of a neighborhood of $\Lambda$, and we can pull back the metric $g$ and the lamination $\mathcal{L}$ to $\pi^{*}(v(\Lambda))$. Inside the pullback lamination, the zero section, which is isometric to $\tilde{\Lambda}$, is an accumulating leave, and is therefore stable by the same argument as above.

Now we have proven that each leaf is either orientable and stable, or has a stable double cover. Therefore Theorem 2.7 applied to balls near each singular point $p_{\infty, j}$ implies that the limit lamination $\mathcal{L}$ has removable singularity, and each leave extends to a smooth, embedded, complete, minimal surface. Pick a leaf $\bar{\Lambda}$ in the extended lamination; by Lemma $4.1, \bar{\Lambda}$ is stable when it is orientable, or has a stable double cover, contradicting the condition $R i c_{g}>0$ via Theorem 4.2.

Remark 3.3 After completing this paper, we realized that [23] proved similar stability result for accumulating leaves using a different method by constructing local calibrations.

Step 3: Suppose that there exists an isolated leaf $\Lambda \in \mathcal{L}$. Then by Step 1 there exists a sequence of minimal surfaces $\left\{\Sigma_{i}\right\}_{i=1}^{\infty} \subset \mathcal{M}_{N}$, so that given any compact subsets $K$ of $\Lambda$, and a tubular neighborhood $U$ of $K$ (such that the intersection of other leaves with $U$ is empty), then $\Sigma_{i} \cap U$ converge locally smoothly to $\Lambda$. In this step, we consider the case when the multiplicity of the convergence is greater than one, and we will postpone the case of multiplicity one convergence to the next step.

By similar argument as in Step 2, we can simply assume that $\Lambda$ is orientable with a unit normal vector field. For $i$ large enough we can choose domains $\Omega_{i} \subset \Lambda$ exhausting $\Lambda$, and tubular neighborhoods $U_{i}$ of $\Omega_{i}$ with $U_{i} \cap \Sigma_{i}=\Omega_{i}$, so that $\Sigma_{i} \cap U_{i}$ decomposes as a multi-valued normal exponential graph over $\Omega_{i}$. By embeddedness and orientability of $\Lambda$, these sheets are ordered by height. Using arguments similar to those in the proof of $[8$, Theorem1.1], we can construct a positive solution $u$ to the Jacobi equation $L u=0$ on $\Lambda$. Therefore $\Lambda$ is stable.

Now there are two possibilities. The simpler case is when $\Lambda$ is properly embedded, and Lemma 2.5 implies that $\Lambda$ has removable singularities, and the closure $\bar{\Lambda}$ is a closed, smooth, embedded, orientable minimal surface, which is stable by Lemma 4.1, contradicting $R i c_{g}>$ 0 . If $\Lambda$ is not properly embedded, we can consider the set-theoretical closure $\bar{\Lambda}$ of $\Lambda$ in $M$, and such a closure forms a sub-lamination $\mathcal{L}_{\Lambda}$ of $\mathcal{L}^{2}$.

Besides $\Lambda$, this sub-lamination contains only accumulating leaves by definition. Following arguments in Step 2, $\mathcal{L}_{\Lambda}$ satisfies the requirement of Theorem 2.7, and has removable singularities. Using arguments in Step 2 again, we can get a contradiction to Ric $c_{g}>0$.

Step 4: Now we focus on the case when the multiplicity of the convergence of $\Sigma_{i}$ to each leaf is one. In this case, we are going to construct a Jacobi field along a leaf, say $\Lambda$. We will show that $\Lambda$ extends to a closed minimal surface with a nontrivial Jacobi field, which violates the bumpy condition.

First we will show that $\Lambda$ has removable singularities. In fact, near each singular point $p \in \bar{\Lambda} \backslash \Lambda$, there is a small radius $r_{p}>0$ such that $\Lambda \cap B_{r_{p}}(p) \backslash\{p\}$ is stable. If this is not

2 Since $\mathcal{L}$ is closed, $\bar{\Lambda}$ is a subset of $\mathcal{L}$ as subsets of $M$. Given any local coordinate chart $(U, \Phi)$ as in Definition 2.2, and an arbitrary point $p \in(\bar{\Lambda} \backslash \Lambda) \cap U$, we claim that the leaf of $\mathcal{L} \cap U$ passing through $p$, i.e. $\Phi^{-1}\left(t_{p}\right)$ where $t_{p}$ is the $t$-coordinate of $\Phi(p)$ in $\Phi(U)$, is contained in $\bar{\Lambda}$, and this implies that $\bar{\Lambda}$ has a lamination structure. To show the claim, let $p_{i} \in \Lambda$ be a sequence of points converging to $p$. Denote $t_{i}$ by the $t$-coordinate of $\Phi\left(p_{i}\right)$ in $\Phi(U)$, then $t_{i} \rightarrow t_{p}$. Therefore $\Phi^{-1}\left(t_{i}\right)$ converge to $\Phi^{-1}\left(t_{p}\right)$ as sets. Hence $\Phi^{-1}\left(t_{p}\right)$ contains all limit points of $\Lambda$, and $\Phi^{-1}\left(t_{p}\right) \subset \bar{\Lambda}$. 
true, by similar arguments as in [15, Proposition1] and [29, §4,Claim2], we can construct infinitely many vector fields with pairwise disjoint compact supports in $B_{r}(p) \backslash\{p\}$ for some $r>0$, along which the second variation of $\Lambda$ are negative. Since the convergence of $\Sigma_{i}$ to $\Lambda$ is locally smooth on $B_{r}(p) \backslash\{p\}$, the second variation of $\Sigma_{i}$ along these vector fields will be negative for $i$ large enough, and this is a contradiction to the fact the $\Sigma_{i}$ has bounded Morse index. The fact that $\Lambda$ has removable singularity follows exactly the same as in Step 3.

We have two possibilities similarly as above. If $\Lambda$ is not properly embedded, we can get a contradiction by looking at the accumulating leaves of the sub-lamination formed by the set-theoretical closure of $\Lambda$. In particular, as in Step $3, \bar{\Lambda} \backslash \Lambda$ is non-empty, and contains all accumulating leaves. Take an accumulating leaf $\Lambda^{\prime} \subset \bar{\Lambda} \backslash \Lambda$, and consider its set-theoretical closure $\overline{\Lambda^{\prime}}$, then this new sub-lamination $\mathcal{L}_{\Lambda^{\prime}}$ of $\mathcal{L}$ contains all stable leaves, and we reduce to a case similar to Step 2 .

Therefore $\Lambda$ is properly embedded, and $\bar{\Lambda}$ is a closed, smooth, embedded, minimal surface. We will construct a nontrivial Jacobi field along $\bar{\Lambda}$ by modifying the proof in $[8$, Theorem1.1andLemmaA.1]. In fact, the key missing point in our case is the lack of a priori uniform area bound for the sequence $\Sigma_{i}$. Theorefore we could not a priori get the varifold convergence of $\Sigma_{i}$, so we can not use the Allard Theorem [2] to derive smooth convergence of $\Sigma_{i}$ to $\bar{\Lambda}$ as in [8]. Our strategy is as follows. In this part, we assume that $\Lambda$ is orientable. By the locally smooth convergence with multiplicity one and the fact that $\Lambda$ is isolated, for $i$ large enough we can choose domains $\Omega_{i} \subset \Lambda$ exhausting $\Lambda$, and tubular neighborhoods $U_{i}$ of $\Omega_{i}$ with $U_{i} \cap \Lambda=\Omega_{i}$, so that $\Sigma_{i} \cap U_{i}$ decomposes as a normal exponential graph over $\Omega_{i}$ of a function $u_{i}$. See Fig. 1. As in [31, equation(7)], $L u_{i}$ vanishes up to higher order terms which converge to zero as $i \rightarrow 0$. Here $L$ is the Jacobi operator of $\Lambda$. Let $h_{i}=u_{i} /\left|u_{i}\right|_{L^{2}\left(\Omega_{i}\right)}$, then the $h_{i}$ 's satisfy uniform local $C^{2, \alpha}$ estimates by similar arguments using elliptic theory as [8, LemmaA.1], and hence converge (up to a subsequence) to a function $h$ on $\Lambda$ locally smoothly with $L h=0$.

It remains to show that $h$ is nontrivial and that $h$ extends smoothly across those discrete points in $\bar{\Lambda} \backslash \Lambda$. To check these two facts, we will show that near each $p \in \bar{\Lambda} \backslash \Lambda$, the $\left|u_{i}\right|$ 's and hence $\left|h_{i}\right|$ 's are bounded by a uniform multiple of their supremum along $\partial B_{\epsilon}(p) \cap \Lambda$ for some small $\epsilon>0$ [see the following (3.2)]. Recall the following facts in the proof of [8, Theorem1.1]. Consider the cylinders $N_{\epsilon}$ (in exponential normal coordinates) over $B_{\epsilon}(p) \cap \bar{\Lambda}$. If $\epsilon$ is small enough, there exists a foliation by minimal graphs $v_{t}$ of some small normal neighborhood of $\bar{\Lambda}$ in $N_{\epsilon}$, so that

$$
v_{0}(x)=0 \quad \text { for all } x \in B_{\epsilon}(p) \cap \bar{\Lambda}, \text { and } v_{t}(x)=t \quad \text { for all } x \in \partial B_{\epsilon}(p) \cap \Lambda,
$$

and $v_{t}$ satisfy uniform Harnack inequality, i.e. $t / C \leq v_{t} \leq C t$ for some $C>0$. Note that we have

Claim $1 \Sigma_{i}$ converge to the limit lamination $\mathcal{L}$ in Hausdorff distance.

Proof Suppose not; then there exist $\delta>0$, and a subsequence of $\Sigma_{i}$ (still denoting by $\Sigma_{i}$ ), such that the intersection of the support of each $\Sigma_{i}$ with $M \backslash U_{\delta}\left(\cup_{\Lambda \in \mathcal{L}} \bar{\Lambda}\right)$ (the complement set of a $\delta$-neighborhood of $\left.\cup_{\Lambda \in \mathcal{L}} \bar{\Lambda}\right)$ is nonempty. However, we know that $\Sigma_{i}$ converge smoothly outside at most $N$ points (on the support of $\mathcal{L}$ ) by Step 1. Also by the monotonicity formula [30, §17], $\Sigma_{i}$ have a uniform area lower bound in $M \backslash U_{\delta}\left(\cup_{\Lambda \in \mathcal{L}} \bar{\Lambda}\right)$. It means that $\Sigma_{i}$ must converge smoothly to a nonempty subset of the lamination $\mathcal{L}$ in $M \backslash U_{\delta}\left(\cup_{\Lambda \in \mathcal{L}} \bar{\Lambda}\right)$, hence a contradiction. 


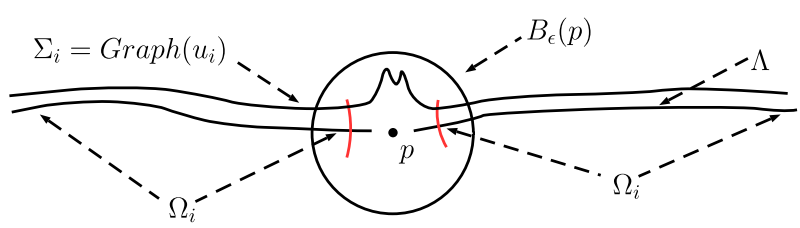

Fig. $1 \Sigma_{i}$ near $p$

Therefore by the Maximum Principle [14, Corollary 1.28] and the Hausdorff convergence, we can follow the argument in the last paragraph of [8, pp119] to show that (see Fig. 1)

$$
\sup _{x \in B_{\epsilon / 2}(p) \cap \Omega_{i}}\left|u_{i}(x)\right| \leq C \sup _{x \in \partial B_{\epsilon}(p) \cap \Lambda}\left|u_{i}(x)\right| .
$$

Now let us go back to check the behavior of $h_{i}$. If the limit of $h_{i}$ is zero, i.e. $h=0$, then $\left|h_{i}\right|$ converge to zero uniformly on any compact subsets of $\bar{\Lambda} \backslash \Lambda$ by the local smooth convergence. In particular $\sup _{\partial B_{\epsilon}(p) \cap \Lambda}\left|h_{i}\right|$ will converge to zero uniformly, and by (3.2), $\sup _{B_{\epsilon / 2}(p) \cap \Omega_{i}}\left|h_{i}\right|$ will also converge uniformly to zero, hence contradicting the fact that $\left|h_{i}\right|_{L^{2}\left(\Omega_{i}\right)}=1$. So $h$ is a nontrivial Jacobi field on $\Lambda$. The same argument can also show that $\sup _{B_{\epsilon / 2}(p) \cap \Omega_{i}}\left|h_{i}\right|$ are uniformly bounded, and hence $h$ extends smoothly across $p$. So $h$ is a nontrivial Jacobi field on $\bar{\Lambda}$, contradicting the bumpy condition, c.f. [34, Theorem2.2].

If $\Lambda$ is non-orientable, we can construct a nontrivial Jacobi field along $\bar{\Lambda}$ similarly by lifting to a double cover. Since $\bar{\Lambda}$ is closed and smooth, by the proof in [37, Proposition3.7], we can construct a double cover $\tilde{M}$ of $M$, such that the pre-image of $\bar{\Lambda}$ (which is also a double cover of $\bar{\Lambda}$ ) is a smooth, embedded, orientable, minimal surface. Denote $\tilde{\Lambda}$ by the pre-image of $\Lambda$ in $\tilde{M}$. Let $\tau: \tilde{M} \rightarrow \tilde{M}$ by the inversion map such that $M=\tilde{M} /\{i d, \tau\}$ and $\bar{\Lambda}=\overline{\tilde{\Lambda}} /\{i d, \tau\}$. Let $\tilde{v}$ be the unit normal vector field of $\tilde{\Lambda}$, then $\tilde{v}$ is anti-symmetric, i.e. $\tau_{*} \tilde{v}=-\tilde{v}$. By counting the sheets of the pre-image $\tilde{\Sigma}_{i}$ of $\Sigma_{i}$ in a small neighborhood of an arbitrary point $\tilde{p} \in \tilde{\Lambda}$ in $\tilde{M}$, which is isomorphic to a small neighborhood in $M$, we can show that $\tilde{\Sigma}_{i}$ converge locally smoothly with multiplicity one to $\tilde{\Lambda}$. Then for $i$ large, $\tilde{\Sigma}_{i}$ can be written as a normal exponential graph over $\tilde{\Lambda}$ of a function $u_{i}$ in the same manner as above.

Claim $2 u_{i}$ is anti-symmetric with respect to $\tau$, i.e. $u_{i} \circ \tau=-u_{i}$.

Proof Fix an arbitrary $p \in \Lambda$, and let $p_{1}, p_{2}$ be the pre-image of $p$ in $\tilde{\Lambda}$. For $i$ large, take $x_{i} \in \Sigma_{i}$ such that the nearest point projection of $x_{i}$ to $\Lambda$ is $p$. Denoting $\tilde{x}_{i}^{1}$ and $\tilde{x}_{i}^{2}$ by the pre-image of $x_{i}$ in $\tilde{M}$, then $\tilde{x}_{i}^{j}=\exp _{p_{j}}\left(u_{i}\left(p_{j}\right) \tilde{v}\left(p_{j}\right)\right), j=1,2$. As $\tilde{v}$ is anti-symmetric and $\tilde{x}_{i}^{1}, \tilde{x}_{i}^{2}$ go to the same point in $M, u_{i}$ must be anti-symmetric.

Theorefore, by working through the proof as above, the sequence of functions $h_{i}=$ $u_{i} /\left|u_{i}\right|_{L^{2}}$ converge to a nontrivial solution $h$ of the Jacobi field equation on $\overline{\tilde{\Lambda}}$, and $h$ is anti-symmetric, i.e. $h \circ \tau=-h$. Then $\tilde{X}=h \tilde{\nu}$ is a symmetric Jacobi vector field on $\overline{\tilde{\Lambda}}$, and hence descends to a nontrivial Jacobi field on $\bar{\Lambda}$, so contradicting the bumpy condition.

Acknowledgments Part of this work was done while the first author was visiting MIT and he wishes to thank MIT for their generous hospitality. The second author wants to thank Toby Colding, Bill Minicozzi and Rick Schoen for helpful conversation on this work. Finally, we thank the referee for very useful comments. 


\section{Appendix}

Here we show that stability can be extended across points on a minimal surface by logarithmic cutoff trick. We add a proof for completeness (see also [20, Proposition1.9]).

Lemma 4.1 Let $\Sigma$ be a smooth minimal surface in $(M, g)$ with trivial normal bundle, and $p$ is an arbitrary point on $\Sigma$. If $\Sigma \backslash\{p\}$ is stable, then $\Sigma$ is stable.

Proof Denote $r$ by the distance function to $p$ on $\Sigma$. Given $\epsilon>0$ small enough, and let $\eta(r)$ be a cutoff function defined by:

$$
\eta(r)=\left\{\begin{array}{l}
0 \quad \text { if } r \leq \epsilon^{2}, \\
\frac{2 \log \epsilon-\log r}{\log \epsilon}, \quad \text { if } \epsilon^{2} \leq r \leq \epsilon, \\
1 \quad \text { if } r \geq \epsilon,
\end{array}\right.
$$

Then $|\nabla \eta| \leq \frac{1}{|\log \epsilon|} \frac{|\nabla r|}{r} \leq \frac{1}{\epsilon^{2}|\log \epsilon|}$. Now given $\varphi \in C^{\infty}(\Sigma)$, by the stability of $\Sigma \backslash\{p\}$,

$$
\int_{\Sigma}\left(|A|^{2}+\operatorname{Ric}(v, v)\right) \eta^{2} \varphi^{2} \leq \int_{\Sigma}|\nabla(\eta \varphi)|^{2} .
$$

The right hand side can be estimated as:

$$
\int_{\Sigma}|\nabla(\eta \varphi)|^{2} \leq \int_{\Sigma} \eta^{2}|\nabla \varphi|^{2}+2 \eta \varphi|\nabla \eta||\nabla \varphi|+\varphi^{2}|\nabla \eta|^{2} .
$$

Therefore,

$$
\int_{\Sigma} 2 \eta \varphi|\nabla \eta||\nabla \varphi| \leq C \int_{\Sigma \cap B_{\epsilon}(p)} \frac{1}{\epsilon^{2}|\log \epsilon|} \leq C^{\prime} \frac{1}{\epsilon^{2}|\log \epsilon|} \epsilon^{2}=\frac{C^{\prime}}{|\log \epsilon|} .
$$

Also denoting $N=|\log \epsilon|$,

$$
\begin{gathered}
\int_{\Sigma} \varphi^{2}|\nabla \eta|^{2} \leq \frac{C}{(\log \epsilon)^{2}} \int_{\Sigma \cap\left(B_{\epsilon}(p) \backslash B_{\epsilon^{2}}(p)\right)} \frac{1}{r^{2}}=\frac{C}{(\log \epsilon)^{2}} \sum_{l=1}^{N} \int_{\Sigma \cap\left(B_{e^{-2 N+l}}(p) \backslash B_{e^{-2 N+l-1}}(p)\right)} \frac{1}{r^{2}} \\
\leq \frac{C^{\prime}}{(\log \epsilon)^{2}} \sum_{l=1}^{N} \frac{1}{\left(e^{-2 N+l-1}\right)^{2}}\left(e^{-2 N+l}\right)^{2}=\frac{C^{\prime} e^{2}}{(\log \epsilon)^{2}} \cdot N=\frac{C^{\prime} e^{2}}{|\log \epsilon|} .
\end{gathered}
$$

Letting $\epsilon$ tend to zero in (4.1), we can get the stability inequality for $\varphi$.

Next we state a result which was essentially due to Fischer-Cobrie and Schoen [16], although it was not stated explicitly there. The proof is well-known to experts, and we include it for completeness.

Theorem 4.2 Let $\left(M^{3}, g\right)$ be a three dimensional Riemannian manifold with positive Ricci curvature, then any complete immersed two-sided minimal surface $\Sigma$ is not stable.

Proof If the result were not true, let $\Sigma$ be stable. We can assume that $\Sigma$ is non-compact. By [16, Theorem3], as $\left(M^{3}, g\right)$ has positive scalar curvature and non-negative Ricci curvature, $\Sigma$ endowed with the pull back metric is conformally equivalent to the complex plane $\mathbb{C}$. Since $\Sigma$ is stable, by [16, Theorem1], there exists a positive function $u$ on $\Sigma$, such that 
$L u=\Delta_{\Sigma} u+\left(|A|^{2}+R i c_{g}(v, v)\right) u=0$, where $L$ is the Jacobi operator (2.1) of $\Sigma$. Since $\operatorname{Ric}(v, v)>0$, we have $\Delta_{\Sigma} u<0$, i.e. $u$ is a superharmonic function. It is well known that any superharmonic function must be constant on $\mathbb{C}$ as $\mathbb{C}$ is parabolic (c.f. [14, Proposition1.37]), hence $\Delta_{\Sigma} u=0$, so we get a contradiction.

\section{References}

1. Anderson, M.: Curvature estimates and compactness theorems for minimal surfaces in 3-manifolds. Ann. Sci. Ec. Norm. Super. IV. Ser. 18, 89-105 (1985)

2. Allard, W.: On the first variation of a varifold. Ann. Math. 95, 417-491 (1972)

3. Choi, H., Schoen, R.: The space of minimal embeddings of a surface into a three-dimensional manifold of positive Ricci curvature. Invent. Math. 81(3), 387-394 (1985)

4. Choi, H., Wang, A.N.: A first eigenvalue estimate for minimal hypersurfaces. J. Differ. Geom. 18, 559-562 (1983)

5. Colding, T., De Lellis, C.: Singular limit laminations, Morse index, and positive scalar curvature. Topology 44(1), 25-45 (2005)

6. Colding, T., Hingston, N.: Metrics without Morse index bounds. Duke Math. J. 119(2), 345-365 (2003)

7. Colding, T., Minicozzi II, W.: Examples of embedded minimal tori without area bounds. Int. Math. Res. Notices 20, 1097-1100 (1999)

8. Colding, T., Minicozzi II, W.: Embedded minimal surfaces without area bounds in 3-manifold. Contemp. Math. 258, 107-120 (2000)

9. Colding, T., Minicozzi II, W.: The space of embedded minimal surfaces of fixed genus in a 3-manifold I; estimates off the axis for disks. Ann. Math. 160, 27-68 (2004)

10. Colding, T., Minicozzi II, W.: The space of embedded minimal surfaces of fixed genus in a 3-manifold II; multi-valued graphs in disks. Ann. Math. 160, 69-92 (2004)

11. Colding, T., Minicozzi II, W.: The space of embedded minimal surfaces of fixed genus in a 3-manifold III; planar domains. Ann. Math. 160, 523-572 (2004)

12. Colding, T., Minicozzi II, W.: The space of embedded minimal surfaces of fixed genus in a 3-manifold IV; locally simply connected. Ann. Math. 160, 573-615 (2004)

13. Colding, T., Minicozzi II, W.: The space of embedded minimal surfaces of fixed genus in a 3-manifold V; fixed genus. Ann. Math. 181(1), 1-153 (2015)

14. Colding, T., Minicozzi II, W.: A course in minimal surfaces. Graduate Studies in Mathematics, vol. 121. American Mathematical Society, Providence, RI (2011)

15. Fischer-Colbrie, D.: On complete minimal surfaces with finite Morse index in three manifolds. Invent. Math. 82, 121-132 (1985)

16. Fischer-Colbrie, D., Schoen, R.: The structure of complete stable minimal surfaces in 3-manifolds of nonnegative scalar curvature. Comm. Pure Appl. Math. 33, 199-211 (1980)

17. Hirsch, M.: Differential Topology, GTM 33. Springer-Verlag, New York (1976)

18. Hass, J., Norbury, P., Rubinstein, J.: Minimal spheres of arbitrarily high Morse index. Comm. Anal. Geom. 11(3), 425-439 (2003)

19. Gulliver, R.: Removability of singular points on surfaces of bounded mean curvature. J. Differ. Geom. 11, 345-350 (1976)

20. Gulliver, R., Lawson, B.: The structure of stable minimal hypersurfaces near a singularity. Proc. Symp. Pure Math. 44, 213-237 (1986)

21. Marques, F.: Minimal surfaces-variational theory and applications. arXiv:1409.7648v1 [math.DG]

22. Marques, F., Neves, A.: Existence of infinitely many minimal hypersurfaces in positive Ricci curvature. arXiv:1311.6501 [math.DG]

23. Meeks, W., Pérez, J., Ros, A.: Limit leaves of an $H$ lamination are stable. J. Differ. Geom. 84, 179-189 (2010)

24. Meeks, W., Pérez, J., Ros, A.: Local removable singularity theorems for minimal laminations. arXiv: $1308.6439 \mathrm{v} 1$

25. Neves, A.: New applications of min-max theory. arXiv:1409.7537v1 [math.DG]

26. Pitts, J.: Existence and Regularity of Minimal Surfaces on Riemannian Manifold, Mathematical Notes 27. Princeton University Press, Princeton (1981)

27. Pitts, J., Rubinstein, J.H.: Applications of minimax to minimal surfaces and the topology of threemanifolds. Proc. CMA 12, 137-170 (1987) 
28. Schoen, R.: Estimates for stable minimal surfaces in three-dimensional manifolds. In: Seminar on minimal submanifolds, pp. 111-126, Ann. of Math. Study, 103, Princeton University Press, Princeton (1983)

29. Sharp, B.: Compactness of minimal hypersurfaces with bounded index. arXiv:1501.02703

30. Simon, L.: Lectures on Geometric Measure Theory. Australian National University Centre for Mathematical Analysis, Canberra (1983)

31. Simon, L.: A strict maximum principle for area minimizing hypersurfaces. J. Differ. Geom. 26, 327-335 (1987)

32. Thurston, W.P.: Three-dimensional geometry and topology. In: Silvio, L. (ed.) Princeton Mathematical Series, 35. Princeton University Press, Princeton, NJ (1997). ISBN: 0-691-08304-5

33. White, B.: Curvature estimates and compactness theorems in 3-manifolds for surfaces that are stationary for parametric elliptic functionals. Invent. Math. 88, 243-256 (1987)

34. White, B.: The space of minimal submanifolds for varying Riemannian metrics. Indiana Univ. Math. J. 40, 161-200 (1991)

35. Yang, P., Yau, S.T.: Eigenvalues of the laplacian of compact Riemann surfaces and minimal submanifolds. Ann. Scuola Norm. Sup. Pisa 7, 55-63 (1980)

36. Yau, S.T.: Problem section. In: Seminar on Differential Geometry, 669-706, Ann. of Math. Stud., 102, Princeton University Press, Princeton (1982)

37. Zhou, X.: Min-max minimal hypersurface in $\left(M^{n+1} ; g\right)$ with $R i c_{g}>0$ and $2 \leq n \leq 6$. J. Differ. Geom. 100, 129-160 (2015) 